- Functional respiratory non-invasive test which mainly showed a decrease in tidal volume with increased respiratory rate volume.

- Non invasive total body plethysmography is an adequate method to study lung function in this animal model.

1316

\section{SERUM YKL-40 AND ASSESSMENT OF ASTHMA SEVERITY IN EGYPTIAN CHILDREN}

T.A. Abd Elgawad, M. Gamal Mansour, M. Abdel-ElAziz, B. Elbialy

Pediatrics, Ain Shams University, Cairo, Egypt

Background: Circulating YKL-40 levels regulated by Polymorphisms in the CHI3L1 (chitinase 3-like 1proteins) gene. This gene represents novel asthma susceptibility gene. Furthermore, circulating YKL40 levels correlated positively with thickening of the lung sub-epithelial basement membrane, frequency of rescue inhaler use, and deterioration in pulmonary function (PF) in European asthmatic ethnics.

Methods: We quantified serum YKL-40 levels in two groups of asthmatics: One group with mild to moderate asthma, and one with severe asthma. Serum YKL-40 was measured by enzyme-linked immunosorbent assay (ELISA) kits (Quidel). Clinical scoring of asthma severity by Pediatric Asthma Score (PAS) and pulmonary functions were performed.

Results: The circulating levels of YKL-40 were significantly elevated in severely asthmatic Egyptian children $(151 \mathrm{ng} / \mathrm{ml})$ compared with the other group (72ng/ml). YKL-40 levelswere correlated significantly to PAS $(r=0.34, p<0.05)$, and to reduced pulmonary function $(r=0.32, p<0.5)$.

Conclusion: YKL-40 is found in increased quantities in the sera of severe asthmatics, and correlated significantly to PAS and PF deterioration. YKL-40 is considered a promising biomarker for asthma severity and pulmonary remodeling warranting further study as a potential novel pathway to disease management.

Abbereviations: $\mathrm{CHI}$ L1; chitinase 3-like 1proteins, PF; pulmonary function, PAS; Pediatric Asthma Score
1317

RAPID DIAGNOSIS OF HAEMOPHILUS INFLUENZA TYPE B PNEUMONIA BY POLYMERASE CHAIN REACTION IN UNVACCINATED EGYPTIAN CHILDREN UNDER FIVE YEARS

M. Mansour, T. Ezzat Deraz, S. Bendary, F. Abdel Moety

Pediatrics, Ain Shams University, Cairo, Egypt

Background: Haemophilus influenza type B (Hib) is an important cause of meningitis,

Community-acquired pneumonia (CAP), and septicemia. Hib disease results in significant morbidity and mortality among children. The burden of Hib pneumonia in Egypt is unclear thus there are no scheduled programs for Hib surveillance, case definition, diagnostic modalities or even vaccination.

Aim: To highlight the magnitude of Hib pneumonia among Egyptian children, and to assess their pattern of antibiotic sensitivity.

Methods: The sera of 100 patients of Egyptian unvaccinated children below five years, presented with community- acquired pneumonia were investigated for Hib pneumonia by Polymerase chain reaction (by ELIZA) and blood cultures.

Antibiotic sensitivity was done for Hib isolates by the use of disc-diffusion method.

Results: Hib pneumonia was diagnosed in 31/100 of patients by PCR patients, 12 of them were culture positive.

The highest resistance of Hib isolates was to cotrimoxazole (75\%) and ampicillin (66.7\%). The highest sensitivity was to ceftriaxon (91.7\%).

PCR had sensitivity of $100 \%$, specificity $74.4 \%$, Positive Predictive value 39\%, Negative Predictive Value $100 \%$ and diagnostic accuracy $81 \%$ compared to blood culture.

Conclusion: The frequency of Hib pneumonia among unvaccinated children in Egypt is alarming.

Combination of PCR and blood culture is necessary to achieve early diagnosis and appropriate antibiotic selection for early diagnosis and better management of Hib pneumonia. 